\title{
CÓDIGO LIVRE PARA SOLUÇÃO NUMÉRICA DAS EQUAÇÕES DE SAINT-VENANT EM CANAIS TRAPEZOIDAIS ASSIMÉTRICOS
}

\author{
OPEN SOURCE FOR NUMERICAL SOLUTION OF SAINT-VENANT EQUATIONS IN \\ ASYMMETRIC TRAPEZOIDAL OPEN-CHANNELS
}

\author{
Italon Rilson Vicente Gamaa, André Luiz Andrade Simõesa, \\ Harry Edmar Schulz ${ }^{b}$, Rodrigo de Melo Portoc
}

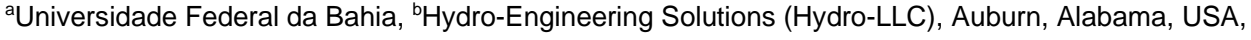
'Escola de Engenharia de São Carlos da Universidade de São Paulo

rilson10@hotmail.com, andre.simoes@ufba.br,.. prof.harryschulz@gmail.com, rodrigo@sc.usp.br.
\end{abstract}

Submissão: 21 de agosto de 2020 Aceitação: 10 de novembro de 2020

\section{Resumo}

Ondas de cheia em canais e ondas produzidas por manobras em comportas são alguns fenômenos simulados com as equações de Saint-Venant em aplicações de engenharia. Um novo código foi desenvolvido para a solução dessas equações aplicadas a um canal trapezoidal assimétrico, empregando o método de volumes finitos de Lax e Friedrichs. Foi adotada uma linguagem de programação reconhecida por um software livre. Três testes numéricos foram realizados. $O$ primeiro, correspondente à passagem de uma onda de cheia em um canal retangular, apresentou aderência aos resultados obtidos com a solução calculada através do método implícito de Preissmann, com desvio relativo máximo de $1,4 \%$ para a velocidade e de $0,81 \%$ para a altura de escoamento. O segundo teste resolveu o escoamento em um canal de fundo variado que induz à formação de um ressalto hidráulico. As comparações dos presentes resultados com aqueles de simulações publicadas recentemente resultaram em um desvio máximo de 2,3\% para as alturas de escoamento, a montante e a jusante do ressalto hidráulico. Para as posições médias do ressalto hidráulico, o desvio foi de $2,4 \%$. Na terceira comparação, simulou-se um ressalto hidráulico em um canal trapezoidal assimétrico de forte declividade, tendo sido encontrada uma solução com desvios relativos menores que $1 \%$ para os escoamentos a montante e a jusante do ressalto, quando comparados aos resultados calculados com o método de MacCormack. A posição média do ressalto nesta terceira comparação apresentou um desvio de 5,5\% em relação aos resultados anteriores. Os desvios calculados indicam que o código desenvolvido é capaz de resolver escoamentos variáveis em canais com e sem a formação de ressaltos hidráulicos. Este é um resultado de cunho prático, pois mostra que códigos livres podem ser usados na prática da hidráulica em geometrias não-convencionais.

Palavras-chave: canais, hidráulica computacional, equações de Saint-Venant.

\section{Abstract}

Flood waves in channels, positive waves produced when operating floodgates, and the hydraulic jump are some phenomena simulated with the Saint-Venant equations in practical engineering applications. A new code was developed to solve these equations applied to an asymmetric trapezoidal channel using the LaxFriedrichs finite volumes method. A programming language recognized by a free software was used. Three numerical tests were performed. The first, corresponding to the passage of a flood wave in a rectangular channel, showed adherence to results of the solution calculated using the Preissmann implicit method, presenting a maximum relative deviation of $1.4 \%$ for the speed and $0.81 \%$ for the flow height. The second 
test solved the flow in a channel with a variable bed that induces the formation of a hydraulic jump. Comparisons of the present results with those of recently published simulations produced a maximum deviation of $2.3 \%$ for the flow heights, upstream and downstream of the hydraulic jump. For the mean positions of the hydraulic jump the deviation was $2.4 \%$. In the third comparison a hydraulic jump was simulated in an asymmetric trapezoidal channel with a strong slope, obtaining a solution with relative deviations less than $1 \%$ for flows upstream downstream of the jump, when compared to the results calculated with the MacCormack method. The average position of the jump in this third comparison showed a deviation of $5.5 \%$ in relation to the former results. The calculated deviations indicate that the developed code is capable of solving variable flows in channels with and without the formation of hydraulic jumps. This is a practical result, because it shows that open codes can be used in the practice of hydraulics in nonconventional geometries.

Keywords: computational hydraulics, open-channel flow, Saint-Venant equations.

\section{INTRODUÇÃO}

Considerando as soluções de problemas próprios da engenharia, os escoamentos em superfície livre podem ser modelados de diferentes formas, a saber: com as equações de conservação de massa, de Navier-Stokes com médias de Reynolds e modelos de turbulência, em duas ou três dimensões, assumindo regime permanente ou regime variável. De forma relativamente mais simples, as equações de Navier-Stokes e de conservação de massa podem ser integradas ao longo da vertical, empregando a abordagem de Saint-Venant, que faz uso de uma distribuição de pressões hidrostática, ou com a abordagem de Boussinesq, que resulta em uma distribuição de pressões diferente da hidrostática (CHAUDHRY, 2008; CASTRO-ORGAZ; HAGER, 2019). Nestes casos, a formulação resultante é bidimensional e relaciona a altura de escoamento $h$ com as coordenadas horizontais $\mathrm{x}$ e $\mathrm{z}$.

Em um terceiro nível de modelagem, são aplicadas as equações de conservação de massa e a segunda lei de Newton a um volume de controle de um escoamento variável assumido como unidimensional e com distribuição de pressões hidrostática. Tais simplificações foram utilizadas pela primeira vez por Saint-Venant (1871), motivo pelo qual as equações diferenciais parciais resultantes recebem o seu nome.

Os modelos mencionados são formados por sistemas de equações diferenciais parciais e equações algébricas, em todos os casos não lineares. Por esse motivo, as soluções exatas ou analíticas são conhecidas apenas para casos simples, sendo necessário o uso de métodos numéricos e códigos para a obtenção de soluções que representem situações mais próximas da realidade da engenharia. Nesse sentido, as literaturas de Hidráulica e Matemática Aplicada contêm elementos comuns sobre o tema, sendo possível citar, por exemplo, os trabalhos dos matemáticos Lax (1954), Lax e Richtmyer (1956), Godunov (1959), Courant et al. (1967) e MacCormack (1969).

Os esquemas numéricos de Lax (1954), Godunov (1959), com diferentes limitadores de fluxo, e MacCormack (1969) foram empregados por Candezano et al. (2011) e Simões et al. (2011), escritos em códigos reconhecidos pelo Matlab $^{\circledR}$, para resolver os problemas clássicos de ruptura de barragem e de formação de ondas de choque (ressalto hidráulico), tendo sido encontrada concordância entre os métodos empregados.

Utilizando as equações de Saint-Venant em 1D e os esquemas numéricos de Lax-Friedrichs e MacCormack, Simões et al. (2017) desenvolveram códigos capazes de simular soluções contínuas, como ondas de cheia em canais trapezoidais, e ondas de choque, como ondas provocadas pela ruptura instantânea de barragens e o ressalto hidráulico. Os referidos autores empregaram a linguagem do software comercial Matlab ${ }^{\circledR}$.

Yu et al. (2019) desenvolveram um modelo de acoplamento entre as equações de SaintVenant em 1D e as equações de Saint-Venant em 2D. Aqueles autores realizaram comparações com outras formas de acoplamento, tendo concluído haver um avanço na qualidade dos resultados numéricos com o método proposto.

Yang et al. (2019) realizaram um estudo 
comparativo entre soluções obtidas com as equações de Navier-Stokes com médias de Reynolds, em duas dimensões, empregando diferentes modelos de turbulência e a solução analítica de Stoker (1957), que é uma solução para as equações de Saint-Venant, considerando escoamento unidimensional um canal retangular largo, horizontal e sem resistência. Os autores utilizaram o software comercial FLOW-3D, tendo encontrado concordância entre as soluções calculadas com os diferentes modelos de turbulência e as posições das descontinuidades previstas pela solução analítica de Stoker (1957).

Em mais um exemplo que demonstra o constante desenvolvimento de pesquisas nesta área, Hodges e Liu (2019) elaboraram e testaram um novo método de volumes finitos para solucionar as equações de Saint-Venant em uma dimensão, incluindo a integração numérica com o método de Runge-Kutta de $4^{\mathrm{a}}$ ordem. $\mathrm{O}$ método numérico proposto foi implementado em Python em um código aberto denominado como SvePy, disponibilizado pelos autores. Foi empregado um teste frequentemente adotado, que consiste em simular o escoamento subcrítico em um canal horizontal seguido de uma elevação de fundo, com consequente mudança de regime e formação de ressalto hidráulico a jusante.

Liu et al. (2020) desenvolveram um modelo hidrodinâmico bidimensional baseado nas equações de águas rasas em $2 \mathrm{D}$ e com um método do tipo Lattice-Boltzmann. Mintgen e Manhart (2018) apresentaram uma formulação para o acoplamento entre as equações de NavierStokes com médias de Reynolds e as equações para o caso de águas rasas, às vezes denominadas como equações de Saint-Venant em 2D, em regime não permanente. Os referidos autores fizeram tal ligação, entre as formulações, através de condições de contorno na interface entre os escoamentos $2 \mathrm{D}$ e $3 \mathrm{D}$, e empregaram o software livre de dinâmica dos fluidos computacional OpenFOAM.

$O$ presente trabalho teve como objetivo $O$ desenvolvimento de um código livre capaz de resolver numericamente as equações de SaintVenant em 1D, para escoamentos em canais trapezoidais assimétricos. O código desenvolvido teve como base aquele proposto por Simões et al. (2017), escrito para o software comercial Matlab ${ }^{\circledR}$, e foi elaborado em linguagem reconhecida pelo software gratuito Scilab.

\section{MATERIAIS E MÉTODOS}

Em 1871, Saint-Venant publicou um sistema de equações deduzidas a partir da conservação de massa e da segunda lei de Newton. A equação (1), originada da equação de conservação de massa, foi apresentada por Saint-Venant (1871) e é válida para as condições de regime não permanente, unidimensional e escoamentos incompressíveis.

$$
\frac{\partial A}{\partial t}+\frac{\partial(u A)}{\partial x}=0
$$

em que $A=$ área molhada $\left(\mathrm{m}^{2}\right), t=$ tempo(s), $u=$ velocidade média $(\mathrm{m} / \mathrm{s})$ na seção transversal e $x(\mathrm{~m})$ a posição ao longo do canal.

O uso da segunda lei de Newton na forma euleriana, aplicada a um volume de controle selecionado em um canal, com uma entrada e uma saída, com paredes indeformáveis e superfície livre deformável, para um escoamento incompressível e turbulento, tem como resultado a equação 2 (PORTO, 2006):

$$
\frac{\partial(u A)}{\partial t}+\frac{\partial}{\partial x}(u A u+g A \bar{h})=g A\left(I_{\circ}-I_{f}\right),
$$

em que $\bar{h}(\mathrm{~m})$ é a profundidade do centro de gravidade da seção transversal, $l_{0}=-\mathrm{d} z / \mathrm{d} x$, sendo $z(\mathrm{~m})$ a cota de fundo, $I_{f}$ é a declividade da linha de energia, grandeza adimensional.

Para obter esta equação, assume-se que o fator de correção da quantidade de movimento seja unitário e que a distribuição de pressões seja hidrostática.

$\mathrm{Na}$ dedução das equações, o somatório de forças inclui uma força decorrente da tensão de cisalhamento, o que origina a perda de carga unitária. Neste trabalho, essa grandeza foi modelada com a equação de Darcy-Weisbach e a equação de Manning, equações 3 e 4 , respectivamente (PORTO, 2006).

$$
I_{f}=f \frac{u[u]}{8 g R_{h}}
$$




$$
I_{f}=n^{2} \frac{u\|u\|}{R_{h}^{4 / a}}
$$

em que $f$ é o fator de resistência de DarcyWeisbach (adimensional), $R_{h}$ é o raio hidráulico (m) e $n$ o coeficiente de Manning $\left(\mathrm{m}^{-1 / 3} \mathrm{~s}\right)$.

As equações 1 e 2 estão escritas na forma conservativa e podem ser substituídas por uma equação vetorial, como indicado na equação 5 (CHAUDHRY, 2008).

$$
\frac{\partial q^{*}}{\partial t}+\frac{\partial f^{*}}{\partial x}=J^{*}
$$

$\mathrm{Na}$ equação $5, q^{*}$ é denominado vetor das quantidades conservadas $e$ possui as componentes $q_{1}=A$ e $q_{2}=u A, f^{*}$ é denominado vetor fluxo e $J^{*}$ é o vetor com os termos fonte. Na forma matricial e empregando as definições de $q_{1}$ e $q_{2}$, escreve-se:

$$
q^{*}=\left(\begin{array}{c}
A \\
u A
\end{array}\right)=\left(\begin{array}{c}
q_{1} \\
q_{2}
\end{array}\right), f^{*}=\left(\begin{array}{c}
u A \\
u A u+g A \bar{h}
\end{array}\right)=\left(\begin{array}{c}
q_{2} \\
\frac{q_{z}^{2}}{q_{1}}+g q_{1} \bar{h}
\end{array}\right) \text { e } I^{*}=\left(\begin{array}{c}
0 \\
g q_{1}\left(I_{o}-I_{f}\right)
\end{array}\right) .
$$

Forma da seção transversal

O modelo computacional elaborado é válido para canais com seção transversal trapezoidal assimétrica e trapezoidal isósceles, canais triangulares e canais retangulares. Essas formas têm área molhada, perímetro molhado e profundidade do centroide que podem ser calculadas a partir do equacionamento geral do trapézio assimétrico, equações 6,7 e 8 , correspondentes à seção transversal apresentada na Figura 1.

\section{Figura 1: Seção transversal trapezoidal assimétrica}

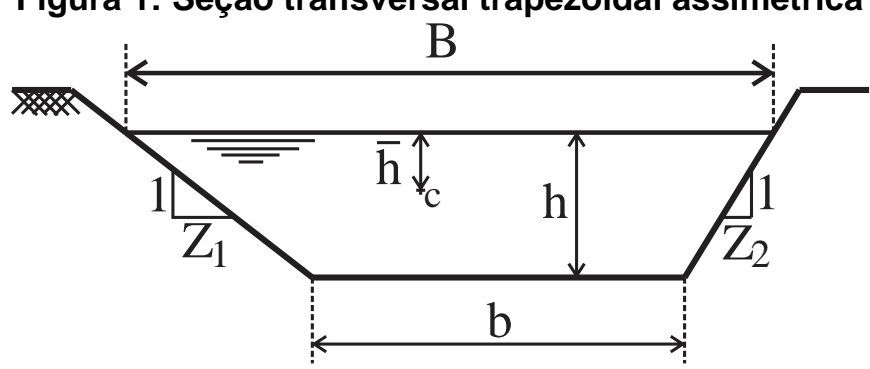

Fonte: próprio autor.

$$
\begin{gathered}
A=\left[2 b+h\left(Z_{1}+Z_{2}\right)\right] h / 2, \\
P=b+h\left(\sqrt{1+Z_{1}^{2}}+\sqrt{1+Z_{2}^{2}}\right), \\
\bar{h}=\frac{h^{2}}{6 A}\left[h\left(Z_{1}+Z_{2}\right)+3 b\right] .
\end{gathered}
$$

$\mathrm{B}$, é necessária para o cálculo do número de Froude, como escrito nas equações 9 e 10 (SIMÕES et al., 2017).

$$
\begin{aligned}
& F r=\frac{u}{\sqrt{g H_{m}}}, \\
& B=b+h\left(Z_{1}+Z_{2}\right),
\end{aligned}
$$

Nestas equações, $Z_{1}$ e $Z_{2}$ são as cotangentes dos ângulos que os taludes esquerdo e direito formam com a horizontal, respectivamente, como na Figura 1 ; b é a largura de fundo e A a área molhada. A largura de topo,

em que $H_{m}=A / B$ é a altura hidráulica.

A solução das equações de Saint-Venant na forma conservativa é $A=A(x, t)$ e $u=u(x, t)$. 
Sendo assim, o cálculo da altura de escoamento a partir da área, empregando a solução analítica para a equação de segundo grau, é realizado com a equação 11 (SIMÕES et al., 2017).

\section{Método numérico}

O método de Lax-Friedrichs foi adotado para a elaboração do código desenvolvido neste trabalho. Lax e Friedrichs propuseram o uso de uma derivada progressiva para a derivada temporal da equação 5 , com o uso de uma média entre os valores de $q^{*}$ dos nós vizinhos à posição considerada. Essa modificação confere estabilidade ao esquema numérico, quando empregada em conjunto com uma derivada espacial centrada, embora exija uma malha refinada para reduzir o efeito difusivo inerente aos métodos de ordem ímpar. A equação 12 expressa a forma implementada no código:

$$
\begin{aligned}
& h=\frac{-b+\sqrt{b^{2}+2\left(Z_{1}+z_{2}\right) A}}{z_{1}+z_{2}} . \\
& q_{i}^{* n+1}=\frac{1}{2}\left(q_{i+1}^{* n}+q_{i-1}^{* n}\right)-\frac{\Delta t}{2 \Delta x}\left[f_{i+1}^{* n}-f_{i-1}^{* n}\right]+\Delta t\left(\frac{j_{1+1}^{* n}+J_{1-1}^{* n}}{2}\right) .
\end{aligned}
$$

Na equação 12, o índice $i$ representa a posição no espaço, ao longo do eixo $x$, o sobrescrito $n$ representa o tempo, $\Delta t$ é o intervalo entre dois instantes do eixo temporal e $\Delta x$ a distância entre dois nós do eixo espacial. $O$ termo fonte foi calculado como uma média, uma das suas possíveis formas de modelagem (CHAUDHRY, 2008). O método de Lax-Friedrichs é condicionalmente estável, devendo-se observar a condição de Courant, equação 13 , para que a estabilidade ocorra.

$$
C n=\frac{\|u\| \pm \sqrt{g H_{m}}}{\Delta x / \Delta t} \leq 1 .
$$

\section{Condições de contorno e condição inicial}

As fronteiras de um canal podem ser de diferentes tipos. Pode existir uma parede impermeável, um vertedor, um orifício, pode ser uma fronteira sobre a qual não temos informações, entre outros casos. Em todos os casos, considerando a natureza hiperbólica das equações de Saint-Venant, deve-se observar que, se ocorre escoamento subcrítico à entrada ou saída, uma variável deverá ser especificada e a outra calculada. Se o escoamento à entrada for supercrítico, as duas variáveis deverão ser especificadas. Se o escoamento de saída for supercrítico, as duas variáveis deverão ser calculadas. Essa regra está fundamentada na análise das curvas características das equações.

A condição inicial corresponde aos valores de $u$ e A em todo o canal no instante $t=0 \mathrm{e}$, também, é dependente do problema estudado. Para a análise de uma abertura de comporta, por exemplo, a água pode estar inicialmente em repouso, assim como para a análise da ruptura instantânea de barragem. Para a simulação de ondas de cheia, por exemplo, um hidrograma deve ser especificado, para a entrada, a altura de escoamento ou área molhada deve ser calculada e, para a saída, utiliza-se a equação de resistência do regime uniforme para o cálculo da altura de escoamento, a partir da vazão calculada com extrapolação de ordem zero. Três testes foram conduzidos com diferentes condições de contorno e iniciais para a realização de comparações entre os resultados calculados com - código proposto e aqueles disponíveis na literatura.

\section{Teste 1: onda de cheia em canal retangular}

Com o intuito de testar o código desenvolvido, foram empregados resultados numéricos obtidos por Porto (2006) e calculados com um código desenvolvido por Graf e Altinakar (1993), o qual resolve as equações de SaintVenant como esquema numérico implícito de Preissmann. O teste realizado comparou as alturas de escoamento, vazões e velocidades nas 
seções inicial, média e final de um canal retangular com $3000 \mathrm{~m}$ de extensão, $5 \mathrm{~m}$ de largura, com $n=0,02$ e $l_{0}=0,001 \mathrm{~m} / \mathrm{m}$. Para a entrada, impõe-se um hidrograma triangular com $Q=8,245 \mathrm{~m}^{3} / \mathrm{s}$ em $t=0, Q=50 \mathrm{~m}^{3} / \mathrm{s}$ em $t=20$ $\min$ e $Q=8,245 \mathrm{~m}^{3} / \mathrm{s}$ em $t=80 \mathrm{~min}$.
Teste 2: fundo variado e formação de ressalto hidráulico em canal retangular

O segundo teste numérico realizado incluiu uma elevação de fundo definida de acordo com a seguinte função, $z=z(x)$ (HODGES; LIU, 2019):

$$
\begin{aligned}
& z(x)=0, \text { para } x<8 \mathrm{~m} \mathrm{e} x>12 \mathrm{~m}, \mathrm{e} \\
& z(x)=0,2-0,05(x-10)^{2}, \text { em }[\mathrm{m}], \text { pata } 8 \leq x \leq 12 \mathrm{~m} .
\end{aligned}
$$

As simulações foram realizadas com 0 código apresentado nos quadros 1 a 4 , com a seguinte adaptação: no lugar de $l_{0}$ como uma constante, foi escrito $l_{0}=-d z / d x$, sendo necessário modificar sua definição computacional de constante para matriz, incluindo os respectivos índices e modificando a componente $\mathrm{J} 2$ do termo fonte. $O$ canal possui seção transversal retangular com $1,0 \mathrm{~m}$ de largura de fundo. A discretização adotada corresponde a um $\Delta t=0,0163 \mathrm{~s}, \Delta x=$ $0,0489 \mathrm{~m}, L=25 \mathrm{~m}$ e tempo total de simulação para estabelecimento do regime permanente igual a $400 \mathrm{~s}$. Para a entrada, foi inserida uma vazão com evolução temporal de 0 a $0,18 \mathrm{~m}^{3} / \mathrm{s}$, em $50 \mathrm{~s}$, e a área molhada foi calculada por extrapolação de ordem zero. Para a saída, fixou-se a altura de escoamento em $0,33 \mathrm{~m}$ e as vazões foram calculadas por extrapolação de ordem zero, já que o escoamento é subcrítico, assim como na entrada.

\section{Teste 3: ressalto e canal trapezoidal assimétrico}

Simões et al. (2017) publicaram um código em Matlab ${ }^{\circledR}$ que resolve as equações de SaintVenant com o método de MacCormack. Em um dos problemas propostos pelos referidos autores, o escoamento é resolvido em um canal trapezoidal com $b=2,5 \mathrm{~m}, I_{0}=0,02 \mathrm{~m} / \mathrm{m}, Z_{1}=$ $1,5, Z_{2}=1,0, Q=5,7 \mathrm{~m}^{3} / \mathrm{s}, n=0,018$ (S.I.), $h(L, t)$ $=2,40 \mathrm{~m}$ e $L=70 \mathrm{~m}$. Os referidos autores simularam a posição do ressalto em $t=600 \mathrm{~s}$. Este terceiro teste, diferente dos dois anteriores, teve como objetivo analisar os resultados calculados com o código proposto quando o canal é trapezoidal e assimétrico $\left(Z_{1} \neq Z_{2}\right)$. Foi empregado $\mathrm{Nx}=300, \mathrm{Nt}=20000$ e número de Courant máximo de 0,65 . A condição de contorno à esquerda corresponde à imposição da altura de escoamento uniforme e da vazão, uma vez que o canal é de forte declividade. Para a saída, foi fixada a altura de escoamento igual a $2,40 \mathrm{~m}$ e a vazão foi calculada com a extrapolação de ordem zero.

\section{Código desenvolvido}

O código elaborado neste trabalho foi desenvolvido para funcionar no software livre e de código aberto Scilab. A sua estrutura básica segue aquela proposta por Simões et al. (2017), indicada para o Matlab $^{\circledR}$, e é composta por: (1) pré-processamento, (2) processamento e (3) pósprocessamento. O Quadro 1 apresenta as linhas do código relativas ao pré-processamento. Com o intuito de facilitar a sua compreensão, foram inseridos comentários em verde, iniciados com //, sendo esta a forma de comentar no Scilab. A condição inicial com a altura de escoamento uniforme foi programada com o método de Newton, em uma função que deve ser colocada na mesma pasta do código. Podem ser empregadas as equações de Manning ou de Darcy-Weisbach. Nas linhas correspondentes, há avisos para que o usuário escreva o local do arquivo da referida função, cujos algoritmos são apresentados no Quadro 2.

O trecho do código que contém as condições de contorno e o método de LaxFriedrichs está no Quadro 3, como etapa de processamento. A condição de contorno de entrada inserida considera a ocorrência de um escoamento subcrítico. Desse modo, a área molhada, $q^{*}$, na posição $\mathrm{i}=1$ (entrada) e em todos os instantes além da condição inicial, é calculada com uma extrapolação de ordem zero. Em termos práticos, essa extrapolação considera que a área molhada no contorno é igual à área molhada no primeiro nó do domínio computacional no instante anterior. Tal estratégia 
é semelhante, conceitualmente, ao obtido com o método das características, mas é algebricamente mais simples, embora não represente prejuízos na precisão do método, como descrito em LeVeque (2007). A vazão é inserida a partir do hidrograma interpolado. Para a saída, uma extrapolação semelhante é empregada para a área molhada. A velocidade é calculada com a equação de Manning ou de Darcy-Weisbach e, em seguida, a vazão é calculada simplesmente como uA.

Prosseguindo com a análise do código, destaca-se 0 trecho que contém o método numérico. Nele, um laço for adicional é empregado para percorrer todos nós internos, ou seja, exceto os nós dos contornos.

\section{Quadro 1: Código em Scilab - Pré-processamento}

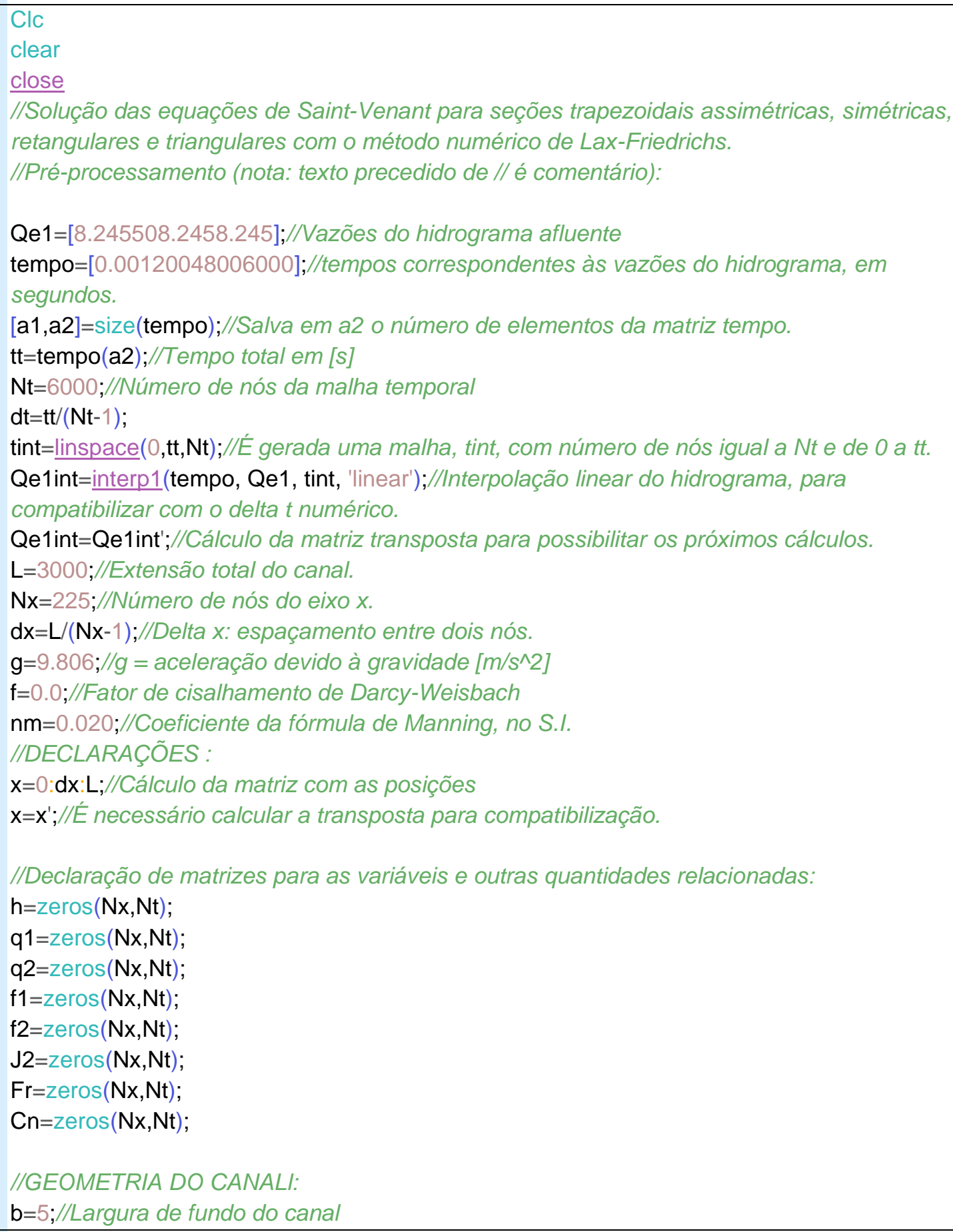


Z1=0;//Co-tangente do ângulo do talude em relação à horizontal (lado esquerdo)

$\mathbf{Z} 2=0 ; / / C o$-tangente (lado direito)

lo=0.0010;//Declividade de fundo

I/CÁLCULO DA CONDIÇÃO INICIAL (h em t=0), empregando o método de Newton em funções externas:

$\mathrm{Q} 0=\mathrm{Qe} 1(1,1) ; / /$ vazão em $t=0$

iff $==0$ then// Se $f=0$, resolve-se a equação de Manning

exec ('cole aqui o local do arquivo, entre aspas simples',-1)//Profundidade do escoamento

uniforme(Manning)

$\mathrm{h} 0=$ uniforme $\mathrm{M}(\mathrm{nm}, \mathrm{Q} 0, \mathrm{~b}, \mathrm{Z1}, \mathrm{Z2}, \mathrm{lo})$

else// Se $n m=0$, resolve-se a equação de Darcy-Weisbach

exec ('cole aqui o local do arquivo, entre aspas simples',-1)

h0=uniforme $\mathrm{DW}(\mathrm{f}, \mathrm{Q} 0, \mathrm{~b}, \mathrm{Z1}, \mathrm{Z2}, \mathrm{lo}, \mathrm{g}) / /$ Profundidade do escoamento uniforme (Darcy-Weisbach) end

INALORES INICIAIS (indicados com um 0 ao lado da variável) ou inseridos para $n=1$ :

$\mathrm{A} 0=\mathrm{b}^{*} \mathrm{~h} 0+0.5^{*}\left(\mathrm{hO}^{\wedge} 2\right)^{*}(\mathrm{Z} 1+\mathrm{Z2}) ; / /$ Área molhada

$\mathrm{u} 0=\mathrm{Q} 0 / \mathrm{A0} ; / /$ Velocidade média

$\mathrm{P} 0=\mathrm{b}+\mathrm{h} 0^{*}\left(\left(1+\mathrm{Z} 1^{\wedge} 2\right)^{\wedge} 0.5+\left(1+\mathrm{Z} 2^{\wedge} 2\right)^{\wedge} 0.5\right)$;//Perímetro molhado

$\mathrm{Rh0}=\mathrm{A0} / \mathrm{P0}$;//Raio Hidráulico

$\mathrm{h}(:, 1)=\mathrm{h} 0 ; / /$ Salva ho em todas as posições e para o instante inicial, $n=1$

$\mathrm{q} 1(:, 1)=\mathrm{A} 0$;

$\mathrm{q} 2(:, 1)=\mathrm{Q} 0$;

$\mathrm{f} 1(:, 1)=\mathrm{q} 2(:, 1)$;

$\mathrm{f} 2(:, 1)=\mathbf{q} 2(:, 1) \cdot{ }^{*} \mathbf{q} 2(:, 1) \cdot / \mathbf{q} 1(:, 1)+(\mathrm{g} / 6)^{*}\left(3^{*} \mathrm{~b}+\mathrm{h}(:, 1) \cdot{ }^{*}(\mathrm{Z} 1+\mathrm{Z} 2)\right) \cdot{ }^{*} \mathrm{~h}(:, 1) \cdot{ }^{\wedge} 2$;

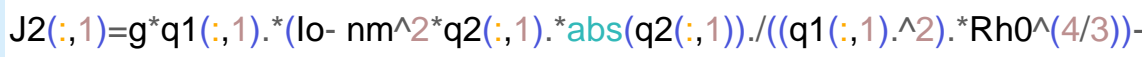

$\left.f^{*} \mathbf{q} 2(:, 1) \cdot{ }^{*} \operatorname{abs}(\mathbf{q} 2(:, 1)) \cdot /\left(\left(\mathbf{q} 1(:, 1) \cdot{ }^{\wedge} 2\right)^{*} 8^{*} \mathbf{g}^{*} \mathrm{Rh} 0\right)\right)$;

$\operatorname{Fr}(:, 1)=\operatorname{abs}(\mathbf{q} 2(:, 1) \cdot / \mathbf{q} 1(:, 1)) \cdot /\left(\left(\mathbf{g}^{*} \mathbf{q} 1(:, 1) \cdot /\left(\mathbf{b}+\mathbf{h}(:, 1) \cdot{ }^{*}(\mathbf{Z 1}+\mathbf{Z} 2)\right)\right) \cdot{ }^{\wedge} 0.5\right) ; / /$ Número de Froude

$\left.\mathrm{Cn}(:, 1)=\left(\operatorname{abs}(\mathbf{q} 2(:, 1) \cdot / \mathbf{q} 1(:, 1))+\left(\mathbf{g}^{*} \mathbf{q} 1(:, 1) \cdot /\left(\mathbf{b}+\mathbf{h}(:, 1)^{*}(\mathbf{Z} 1+\mathbf{Z} 2)\right)\right)\right)^{\wedge} 0.5\right) \cdot /(\mathbf{d x} / \mathbf{d t}) ; / /$ Número de Courant

I/AQUI TERMINA A IMPOSIÇÃO DAS CONDIÇÕES INICIAIS

//GRÁFICO COM O HIDROGRAMA DE ENTRADA

plot(tint/60, Qe1int, 'r',tempo/60, Qe1,'o'); //r escolhe a cor vermelha e 'o' o marcador

xlabel('t [min]');

ylabel $\left(' \mathrm{Q}=\mathrm{uA}\left[\mathrm{m}^{\wedge} 3 / \mathrm{s}\right]\right)$;

Leg=legend('H_2(0,t) - Interpolado','H_2(0,t) - Tabela 1');

scf(1)//função que mantém o gráfico aberto.

//GRÁFICO COM O AS PROFUNDIDADES EM $t=0$

$\operatorname{plot}\left(x, h(:, 1),{ }^{\prime} \circ\right)$;

xlabel('x [m]');

ylabel $(' h(x, 0)[m] ')$;

$\operatorname{scf}(2)$

Fonte: próprio autor. 
Quadro 2: Código em Scilab - Método de Newton para o regime uniforme

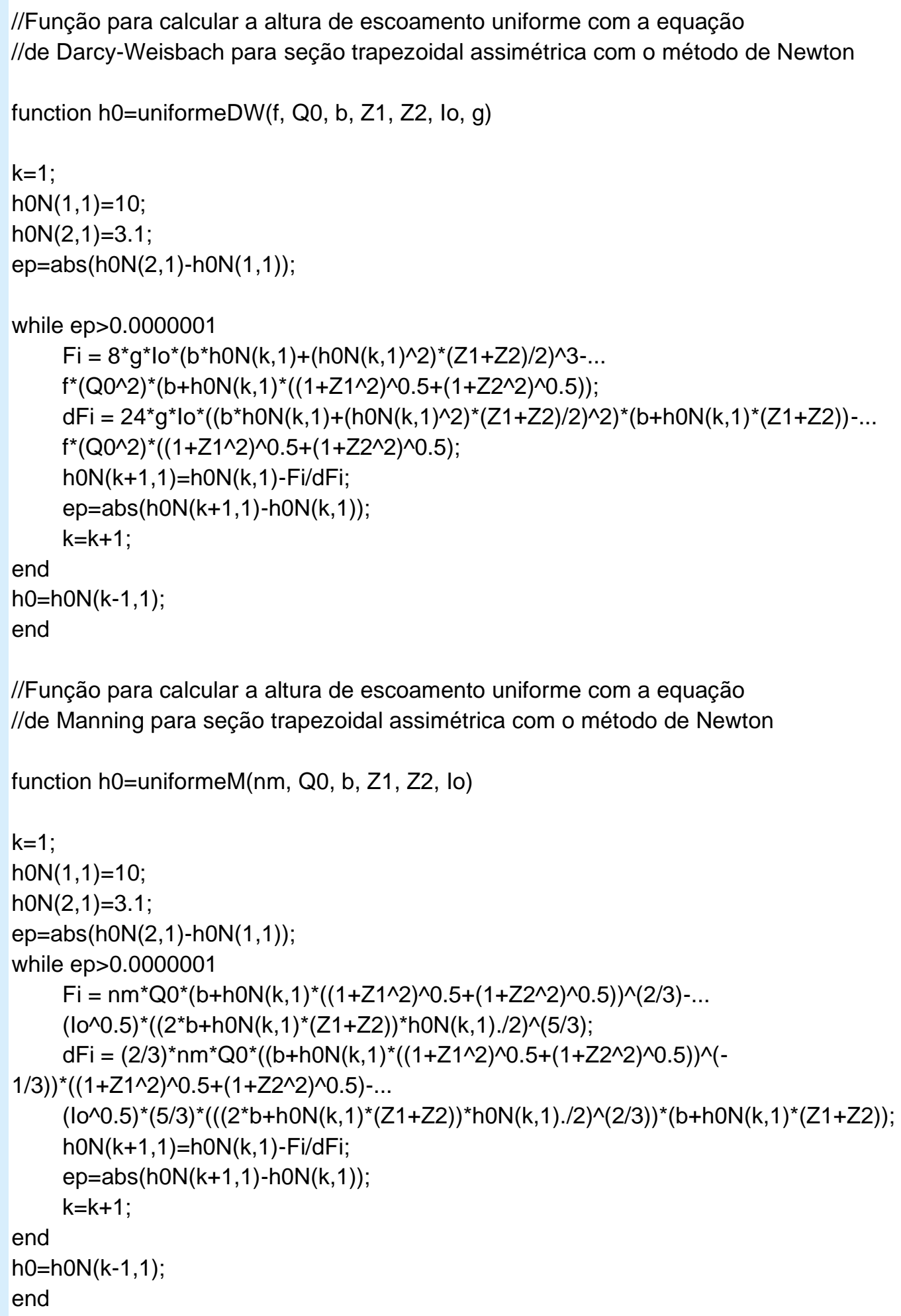

Fonte: próprio autor. 
Quadro 3: Código em Scilab - Processamento

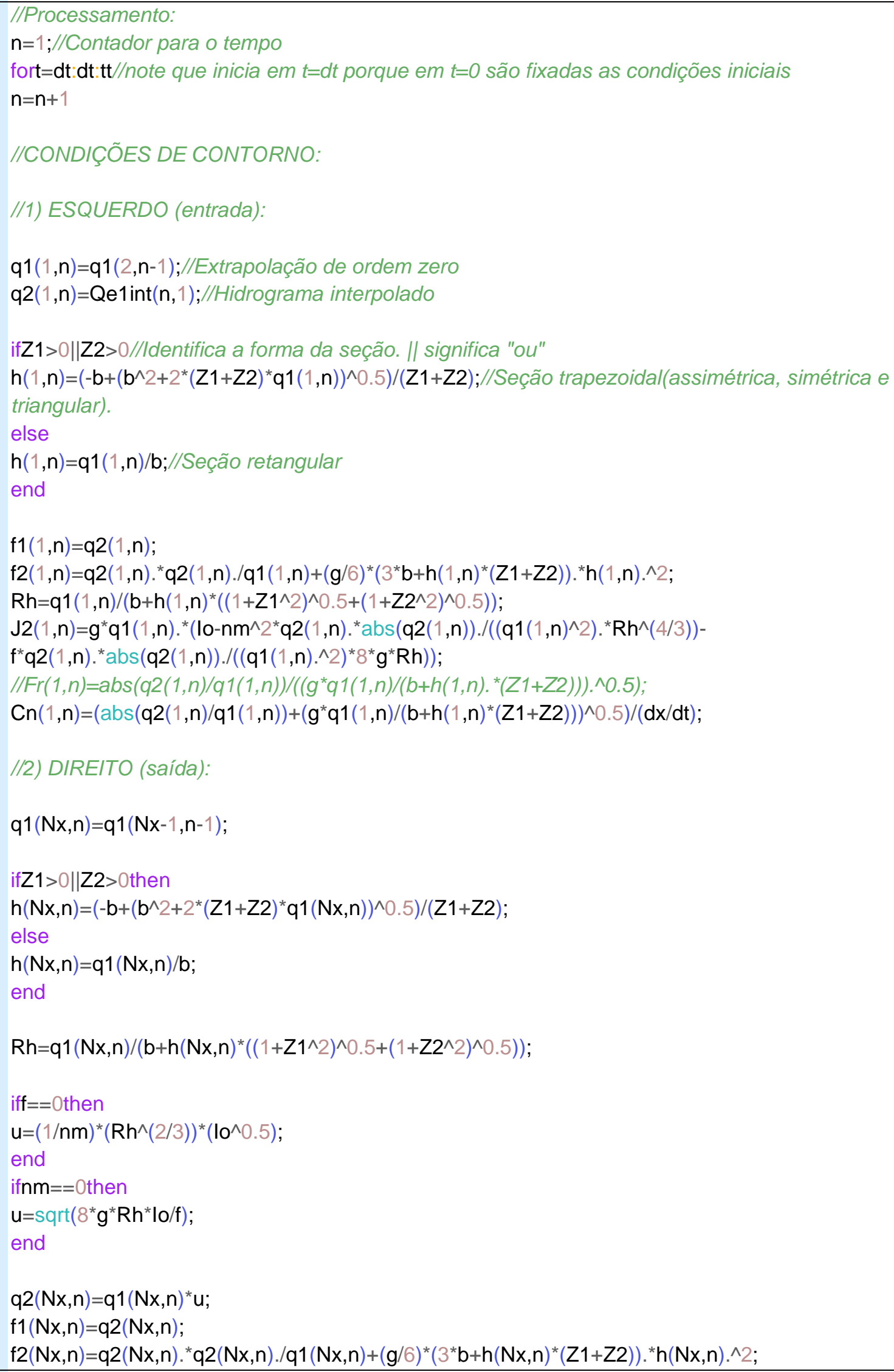




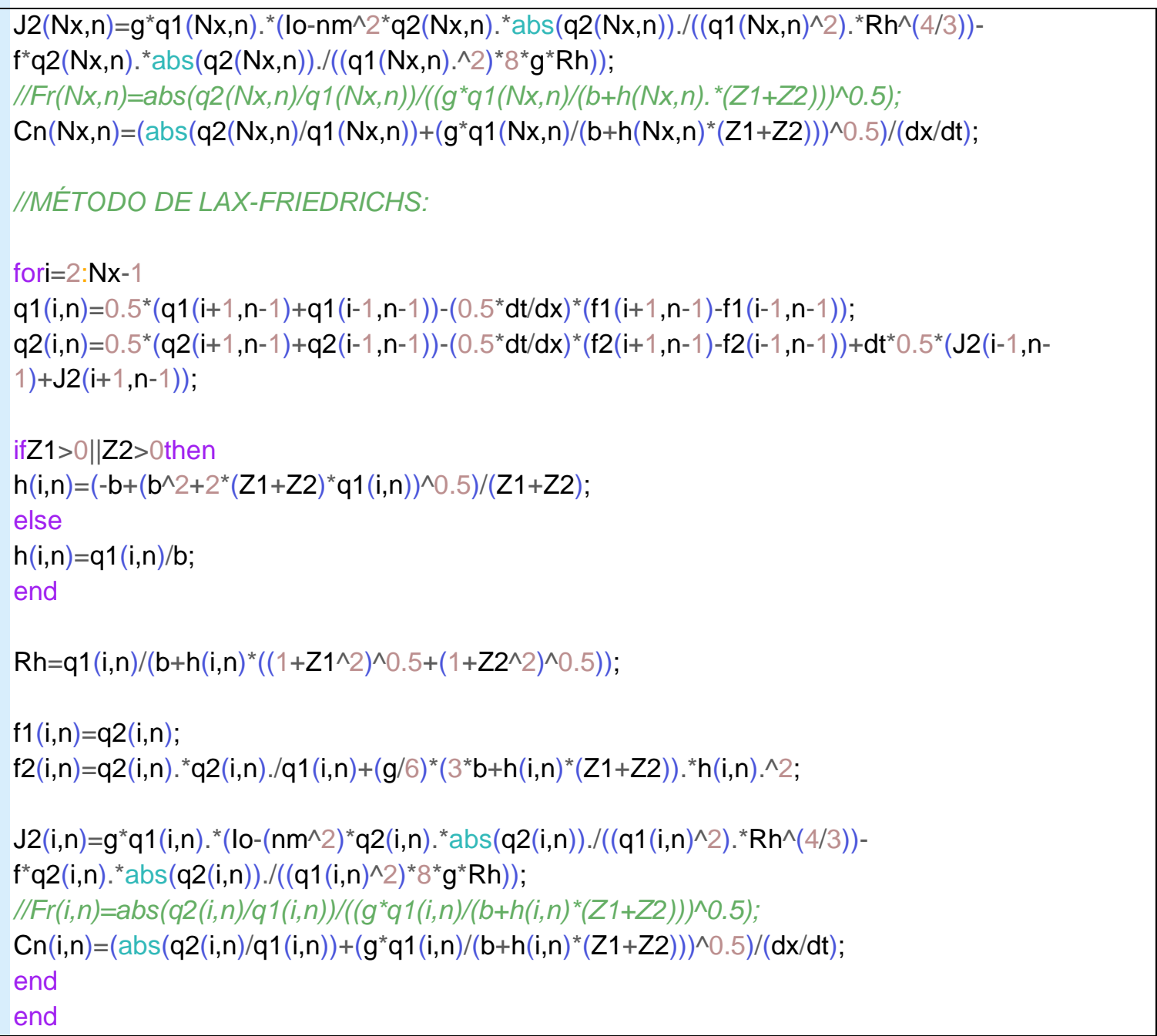

Fonte: próprio autor.

O pós-processamento pode ser explorado com diferentes recursos do Scilab. Entre eles, o Quadro 4 contém códigos escritos para gerar gráficos de $h(x, t), Q(x, t)$ e $u(x, t)$ para $x=0, L / 2$ e L. É apresentado também o código que emprega a função do regime permanente e uniforme para gerar a curva que relaciona a altura de escoamento com a vazão, com o intuito de comparar essa curva com a curva chave em forma de laço do regime variável.

\section{RESULTADOS E ANÁLISE}

Teste 1: onda de cheia em canal retangular

Os resultados obtidos, apresentados na Figura 2, mostram que houve aderência entre as soluções numéricas calculadas com o código proposto e a solução apresentada em Porto
(2006), obtida com o esquema numérico de Preissmann. Para o valor máximo de $h$, em $x=0$ (Fig. 2a), o desvio relativo entre as soluções foi de $0,62 \%$. Para o valor máximo de $Q$, em $x=L / 2$ (Fig. 2b), o desvio relativo máximo resultou igual a $0,81 \%$ e, comparando a velocidade máxima em $x=0$ (Fig. 2c), o desvio relativo resultou igual a $1,4 \%$. O gráfico da Figura $2 d$ contém a relação entre a vazão e a altura de escoamento na seção localizada em $x=L / 2$. Observa-se concordância entre a solução calculada no presente trabalho e aquela calculada por Porto (2006).

Teste 2: fundo variado e formação de ressalto hidráulico em canal retangular

A Figura 3 relaciona a altura de escoamento com a posição ao longo do canal, obtida com número de Courant máximo igual a 1,0. Foram inseridos os resultados numéricos 
obtidos por Hodges e Liu (2019). É possível notar aderência entre os métodos quando considerada a posição da superfície livre a montante e a jusante do ressalto hidráulico. Para esses trechos, o desvio máximo entre os resultados obtidos no presente trabalho e aqueles de Hodges e Liu (2019) foi de 2,3\%, em $x=11,39 \mathrm{~m}$. Para o $\Delta x$ adotado com o código proposto, notase que o efeito difusivo do método de Lax-
Friedrichs resultou em um desvio de $2,4 \%$ da posição média do ressalto hidráulico, que ficou situado entre $x=11,39 \mathrm{~m}$ e $x=12,13 \mathrm{~m}$, enquanto que, com o método de Hodges e Liu (2019), o ressalto ficou entre $x=11,39 \mathrm{~m} \mathrm{e} x=$ 11,59 m. A posição média foi empregada nesta comparação porque ressaltos hidráulicos calculados com as equações de Saint-Venant são descontinuidades.

\section{Quadro 4: Código em Scilab - Pós-processamento}

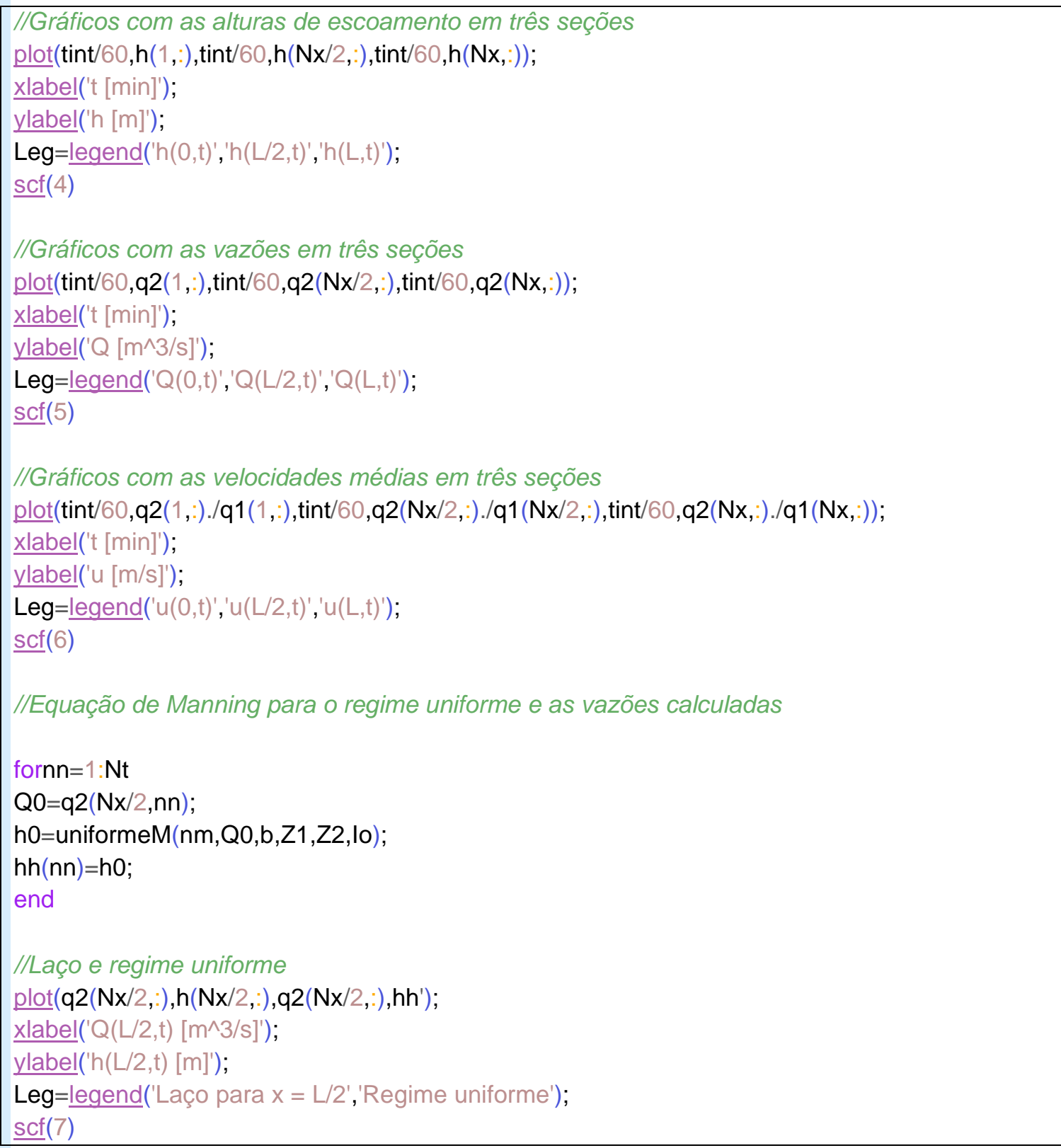

Fonte: próprio autor. 
Figura 2: Solução para uma onda de cheia em um canal retangular: comparação com o código de Porto (2006): (a) altura de escoamento; (b) vazões; (c) velocidades; em todos os casos, nas seções em $x=0, x=L / 2$ e $x=L$. (d) curva chave para a seção em $x=\mathrm{L} / 2$
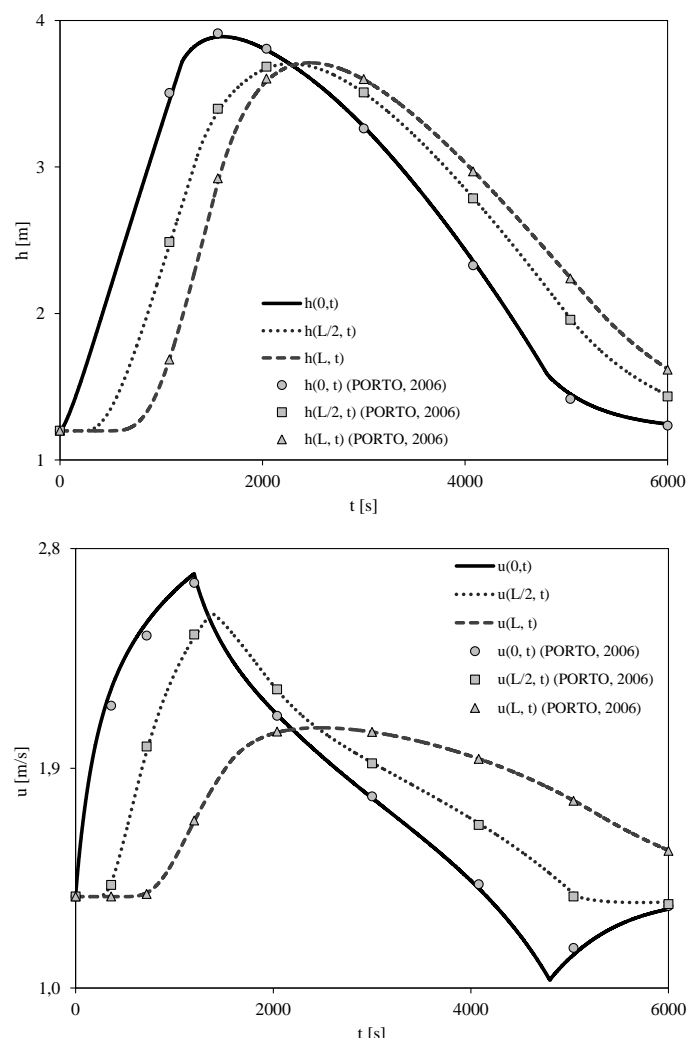

$\mathrm{t}[\mathrm{s}]$

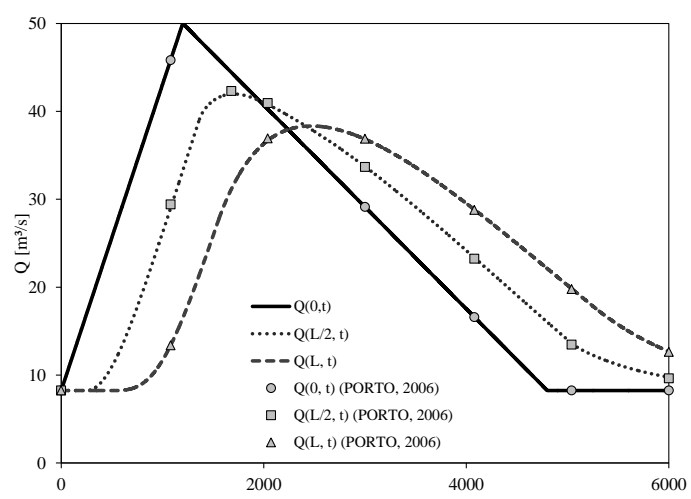

(a)

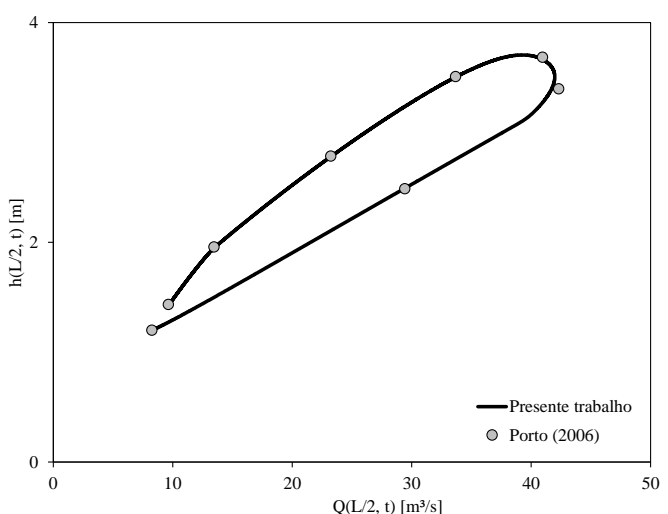

(d)

(c)

(b)

Fonte: próprio autor.

Figura 3: Teste com formação de ressalto hidráulico

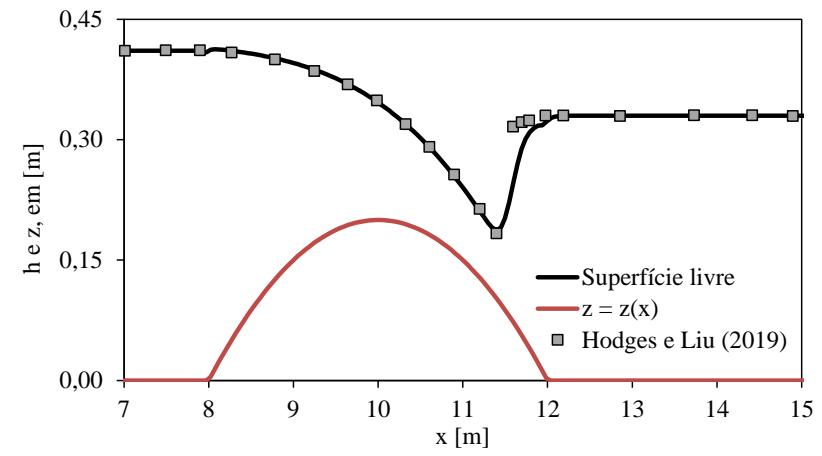

Fonte: próprio autor

Teste 3: ressalto e canal trapezoidal assimétrico

Os resultados obtidos para a posição da superfície livre calculados no presente trabalho encontram-se próximos aos resultados calculados por Simões et al. (2017), como pode ser visto na Figura 4. O desvio relativo entre as soluções para o trecho supercrítico resultou em $0,27 \%$ para a altura de escoamento em $x=2,19 \mathrm{~m}$. Para $x=$ $12,8 \mathrm{~m}$, no trecho subcrítico do escoamento, o desvio relativo foi de $0,87 \%$ entre a solução obtida neste trabalho e aquela apresentada por Simões et al. (2017), com o método de MacCormack. As posições inicial e final do 
ressalto hidráulico, obtidas com 0 código proposto, foram $x=3,28 \mathrm{~m}$ e $x=7,16 \mathrm{~m}$, respectivamente, resultando na posição média $x=$ $5,22 \mathrm{~m}$. A posição média obtida com os dados de
Simões et al. (2017) foi de 4,95 m. Com essas informações, foi calculado o desvio relativo, que resultou em $5,5 \%$.

Figura 4: Ressalto em canal trapezoidal assimétrico

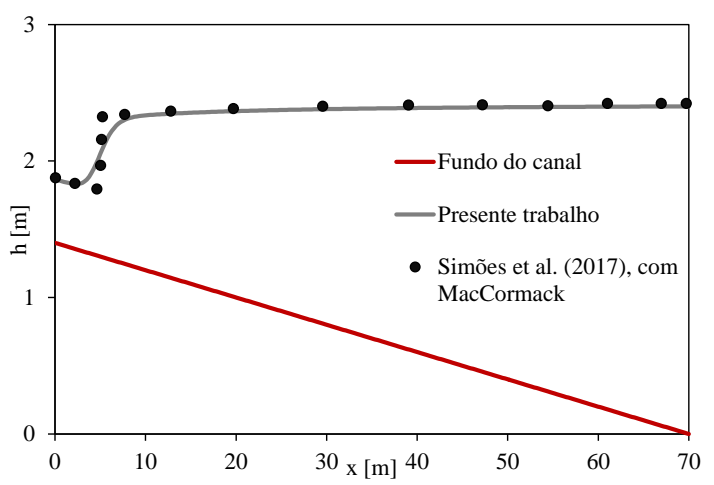

Fonte: próprio autor.

\section{CONCLUSÕES}

A realização deste trabalho teve como resultado a criação de um código gratuito para a simulação de escoamentos não permanentes e não uniformes em canais trapezoidais assimétricos, empregando as equações de SaintVenant e o método numérico de Lax e Friedrichs, em linguagem reconhecida pelo software livre Scilab. O código foi testado com a simulação de uma onda de cheia em um canal retangular, tendo apresentado aderência aos resultados simulados por um segundo código, disponível na literatura e que emprega um método numérico implícito de Preissmann. Em um segundo teste, cuja natureza é mais exigente devido à mudança de regime, formação de ressalto hidráulico e declividade de fundo variada, foram obtidos resultados fisicamente consistentes, ou seja, com ocorrência de escoamento subcrítico a montante da elevação de fundo, com escoamento crítico sobre a crista da elevação de fundo seguido de escoamento supercrítico e ressalto hidráulico. A comparação realizada entre os resultados obtidos com o código proposto e aqueles simulados empregando um método numérico de volumes finitos, publicado recentemente, resultou em desvio relativo máximo para a altura de escoamento igual a $2,3 \%$ e um desvio relativo de $2,4 \%$ para a posição média do ressalto hidráulico.
Um terceiro teste foi realizado para a geometria trapezoidal assimétrica da seção transversal. Neste teste, foi simulado o escoamento com formação de ressalto hidráulico em um canal de forte declividade, e os resultados foram comparados com aqueles disponíveis na literatura obtidos com o método de MacCormack, que é de segunda ordem. A comparação entre as posições da linha d'água indicou haver um desvio relativo máximo de $0,87 \%$. A posição média do ressalto foi calculada, tendo apresentado um desvio relativo de $5,5 \%$ em relação ao método citado. Essas comparações indicam que o código desenvolvido com o método de Lax-Friedrichs é capaz de resolver problemas de forma adequada, incluindo soluções suaves, como as ondas de cheia, e soluções que contêm singularidades, como o ressalto hidráulico. Adicionalmente, um código livre (gratuito) com este grau de complexidade é um resultado prático relevante na atividade profissional da hidráulica.

\section{Agradecimentos}

Os autores agradecem ao apoio da Pró-Reitoria de Ensino de Pós-Graduação da Universidade Federal da Bahia pela bolsa de Iniciação Científica do primeiro autor. 


\section{REFERÊNCIAS}

CASTRO-ORGAZ, O.; HAGER, W.H. Shallow water hydraulics. New York: Springer International Publishing, 2019.

CHAUDHRY, M.H. Open-channel flow. New York: Springer International Publishing, 2008.

CANDEZANO, M.A.C.; SARTORI, P.; SIMÕES, A.L.A.; FERREIRA, V.G. Numerical solutions of shallow water equations with source term using high-resolution bounded upwind schemes. In: CONFERÊNCIA BRASILEIRA DE DINÂMICA, CONTROLE E APLICAÇÕES - Dincon, 10., 2011, Águas de Lindóia. Anais... Águas de Lindóia-SP: SBMAC, 2011.

COURANT, R.; FRIEDRICHS, K.; LEWY, H. On the partial difference equations of mathematical physics. IBM Journal, v. 11, n.2, p. 215-234, 1967. (English translation of the 1928 German original).

GODUNOV, S.K. A difference method for numerical calculation of discontinuous solutions of the equations of hydrodynamics. Math. Sbornik, v. 47, p. 271-306, 1959. (translated US Joint Publ. Res. Service, JPRS 7226, 1969).

GRAF, W.H.; ALTINAKAR, M.S. Hydraulique Fluviale - Tome 2: Ecoulement non permanente et phénomènes de transport. Lausane: Presses Polytechniques et Universitaires Romandes, 1993. 378p.

HODGES, B.R.; LIU, F. Timescale interpolation and noneighbour discretization for a 1D finite-volume SaintVenant solver. Journal of Hydraulic Research, v. 58, n. 5, p. 1-17, 2019. DOI:

10.1080/00221686.2019.1671510

LAX, P.D. Weak solutions of nonlinear hyperbolic equations and their numerical computation. Comm. Pure Appl. Math., n. 7, p. 159-193, 1954.

; RICHTMYER, R.D. Survey of the stability of linear finite difference equations. Communications on Pure and Applied Mathematics, v 9, n. 2, p. 267-293, 1956.

LEVEQUE, R. Finite volume methods for hyperbolic problems. United Kingdom: Cambridge University Press, 2007.
LIU, H; XING, L.; CHEN, X. Lattice Boltzmann method for shallow water flow with wave radiation stress.

Journal of Hydraulic Research, 2020. DOI: 10.1080/00221686.2020.1729264

MACCORMACK, R.W. The effect of viscosity in hypervelocity impact cratering. AIAA paper 69-354, 1969.

MINTGEN, F.; MANHART, M. A bi-directional coupling of 2D shallow water and 3D Reynolds-averaged Navier-Stokes models. Journal of Hydraulic Research, v. 56, n. 6, p. 771-785, 2018. DOI: 10.1080/00221686.2017.1419989

PORTO, R.M. Hidráulica básica. São Carlos: EESCUSP, 2006.

SAINT-VENANT, A.J.C.B. Théorie du movement non permanent des eaux, avec application aux crues de riviéres et à l'introduction des marées dans leur lit. Comptes Rendus des séances de l'Académie des Sciences, Paris, n. 73, p. 147-154, jul.1871.

SIMÕES, A.L.A.; CANDEZANO, M.A.C.; SCHULZ, H. E. ; PORTO, R. M.; FERREIRA, V.G. Comparação de esquemas numéricos para as equações de SaintVenant usando códigos livres. SIMPÓSIO BRASILEIRO DE RECURSOS HÍDRICOS, 19., 2011, Maceió. Anais... Maceió-AL: ABRHidro.

SIMÕES, A.L.A.; SCHULZ, H. E.; PORTO, R. M. Método computacionais em hidráulica. Salvador: EDUFBA, 2017.

STOKER, J.J. Water waves. New York, NY: Interscience, 1957.

YANG, S.; YANG, W.; QIN, S.; LI, Q. Comparative study on calculation methods of dam-break wave. Journal of Hydraulic Research, v.57, n. 5, p. 702-714, 2019. DOI: $10.1080 / 00221686.2018 .1494057$

YU, K; CHEN, Y.; ZHU, D.; VARIANO, E.A.; LIN, J.(2019) Development and performance of a 1D-2D coupled shallow water model for large river and lake networks. Journal of Hydraulic Research, v. 57, n. 6 , p. $852-865,2019$. DOI: 10.1080/00221686.2018.1534286 